University of Nebraska - Lincoln

DigitalCommons@University of Nebraska - Lincoln

7-15-1993

Symmetry selection rules for vibrational excitation by resonant electron impact and a unified treatment of vibronic coupling between resonances and to the continuum: A complete symmetry analysis of vibrational excitation in benzene

Gordon A. Gallup

University of Nebraska-Lincoln, ggallup1@unl.edu

Follow this and additional works at: https://digitalcommons.unl.edu/physicsgallup

Part of the Physics Commons

Gallup, Gordon A., "Symmetry selection rules for vibrational excitation by resonant electron impact and a unified treatment of vibronic coupling between resonances and to the continuum: A complete symmetry analysis of vibrational excitation in benzene" (1993). Gordon Gallup Publications. 3.

https://digitalcommons.unl.edu/physicsgallup/3

This Article is brought to you for free and open access by the Research Papers in Physics and Astronomy at DigitalCommons@University of Nebraska - Lincoln. It has been accepted for inclusion in Gordon Gallup Publications by an authorized administrator of DigitalCommons@University of Nebraska - Lincoln. 


\title{
Symmetry selection rules for vibrational excitation by resonant electron impact and a unified treatment of vibronic coupling between resonances and to the continuum: A complete symmetry analysls of vibrational excitation in benzene
}

\author{
G. A. Gallup \\ Department of Chemistry, University of Nebraska-Lincoln, Lincoln, Nebraska 68588-0304
}

(Received 28 September 1992; accepted 31 March 1993)

\begin{abstract}
A symmetry analysis of the contributions to the transition operator from vibronic coupling provides symmetry selection rules for vibrational excitation by resonant electron impact in polyatomic molecules. In the context of electron scattering vibronic coupling operates in two modes. These are named internal and external vibronic coupling. Internal vibronic coupling operates within and among the quasibound states giving rise to the resonances. External vibronic coupling operates between the quasibound states and the continuum. We discuss the differences, particularly with respect to the angular distribution of electron scattering. A complete symmetry analysis of vibrational excitation is given using benzene as an example.
\end{abstract}

\section{INTRODUCTION}

Experimental results for electron impact excitation of vibration on several polyatomic molecules show conclusively that symmetry mediated selection rules operate. Table I shows those polyatomic molecules known to the author for which experimental determinations of both vibrational excitation functions and their angular distributions exist. ${ }^{1-10}$

On the theoretical side, this author has published a discussion of such selection rules in what will hereinafter be called $\mathrm{I}^{11}$ In I the specific term "vibronic coupling" did not appear, but its use would have been appropriate. Specifically I discusses how vibronic coupling between a resonant state and the continuum can cause excitations of selected vibrations and produce unexpected angular distributions of nearly $s$-wave character.

About the same time and independently, Estrada et al.,$^{12}$ published a model study of vibronic interaction between two resonances. Their work shows how overlapping resonances result in the phenomenon sometimes called the pseudo-Jahn-Teller effect. They do not discuss the case of a degenerate resonant state.

More recently, Ben Arfa and Tronc ${ }^{13}$ have given what they imply is an alternative explanation of the excitation of particular vibrational modes by resonant electron impact. They base their analysis on Estrada et $a .^{12}$ Indeed, the Ben Arfa and Tronc article contains the phrase "Symmetry selection rules vs. vibronic coupling..." in its title. As we shall see, vibronic coupling is the mechanism in all cases.

In the present article we will give a unified treatment of the symmetry considerations for vibronic coupling and resonant excitation of vibrations in polyatomic molecules. We will show that vibronic coupling between resonances and vibronic coupling to the continuum are two aspects of the same phenomenon. In their article Estrada et al. ${ }^{12}$ concentrate much of their discussion on the effects of vibronic coupling upon angular distributions, giving, in detail, cal- culations of a model system. Nevertheless, their treatment implicitly contains the effects giving rise to the resonancecontinuum vibronic coupling discussed in I. In the present article we will again concentrate on the symmetry properties of the various mechanisms, presenting the theory in a form designed to simplify, insofar as possible, its application to real molecules. This will require approximations, but symmetry analyses are not affected by the approximations we use.

\section{VIBRONIC COUPLING}

As we shall use the term, vibronic coupling (VC) will refer to any interaction between the electronic and nuclear motions of a molecule. In electronic spectra of molecules, it has long been recognized that VC can modify selection rules and cause the appearance of spectral transitions that would appear to be disallowed when a simplified stationary nucleus approximation is applied. Many different workers have contributed to this field with a resulting plethora of notations and terminologies. Our terminology will follow the suggestions of Azumi and Matsuzaki, ${ }^{14}$ as further elaborated by Fischer. ${ }^{15}$ They, in particular, discuss the types of bases that one uses to generate the matrix of the Hamiltonian. They distinguish two cases: the crude adiabatic basis and a basis used for two closely similar treatments, the Born-Oppenheimer adiabatic and the Born-Huang adiabatic. It must be emphasized that either of these two bases is a complete set and can, in principle, produce exact answers in the limit.

We base our analysis on the crude adiabatic basis. Selection rules are usually expressed in terms of idealized states upon which the most detailed level of possible perturbations have not been applied. The crude adiabatic basis is more suitable for this since both the electronic and vibrational factors in the basis functions have the full symmetry of the most symmetric equilibrium nuclear arrangement. This greatly simplifies the symmetry analysis. 
TABLE I. Polyatomic molecules for which experimental data on resonant vibrational excitation and angular distributions are available.

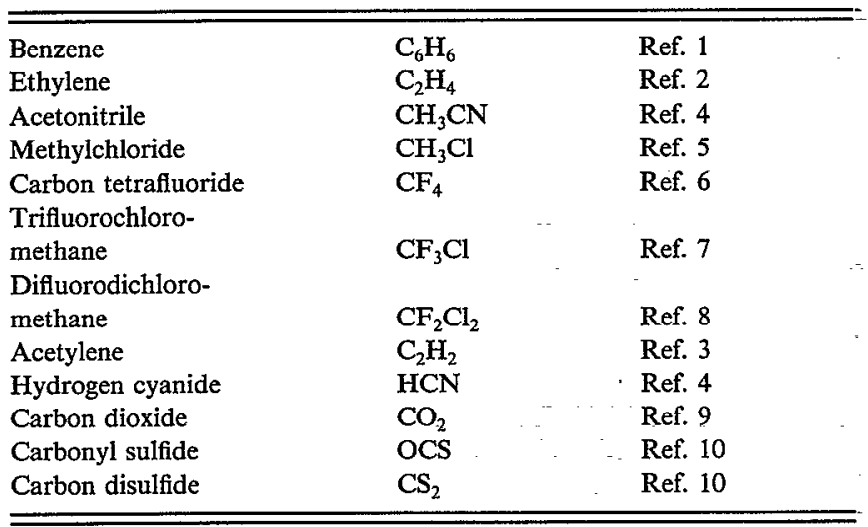

\section{THE TRANSITION MATRIX}

In I the author used a perturbation treatment based upon an $R$-matrix representation of the electronic states. In this article a fully equivalent approach based upon the transition operator will be used. This follows more closely the treatment by Estrada et al., ${ }^{12}$ and provides a clearer comparison with that work.

The Lipmann-Schwinger equation for the transition operator is

$$
T=V+V G_{0} T,
$$

where

$$
\begin{aligned}
& H=H^{0}+V, \\
& G_{0}=\left(E+i 0-H^{0}\right)^{-1} .
\end{aligned}
$$

The Hamiltonian may be subjected to a projection separating the basis into a subspace representing quasibound (resonant) states and the complementary subspace, representing the asymptotic states. Feshbach ${ }^{16}$ and Fano ${ }^{17}$ have shown how to solve the problem of the interaction. This formulation has recently been reviewed by Domcke. ${ }^{18}$ Us:ing subscripts $a$ and $b$ to indicate the asymptotic and resonant states, respectively, the Hamiltonian takes the form

$$
H=\left[\begin{array}{ll}
H_{a a} & H_{a b} \\
H_{b a} & H_{b b}
\end{array}\right],
$$

and the open channel block of the transition operator the form

$$
\begin{aligned}
& T_{a a}=H_{a b}\left[E-H_{b b}-F_{b b}(E)\right]^{-1} H_{b a}, \\
& F_{b b}(E)=H_{b a}\left(E+i 0-H_{a a}\right)^{-1} H_{a b} .
\end{aligned}
$$

The Feshbach-Fano treatment assumes a more or less arbitrary separation of the wave function into an inner region part and an outer continuum part. The arbitrariness is in the dynamical nature of these functions. The symmetries must match and cannot be arbitrary. In an exact treatment the resulting scattering wave function should be invariant to the mode of separation. Actual calculations of an approximate nature show that the results are quite insensitive to the mode of separation. ${ }^{19}$

\section{BASIS SETS AND THE HAMILTONIAN MATRIX}

Our proposed symmetry analysis requires a suitable basis; as discussed abovc, wc use the crude adiabatic representation. Consider a neutral molecule in a singlet ground state. It will have a number of vibrational modes that we approximate at the harmonic level. Using $r$ for the electronic coordinates and $Q$ for the normal coordinates, the Hamiltonian may be written in the form

$$
\begin{aligned}
& H=T(r)+T(Q)+V(r, 0)+\Delta V(r, Q), \\
& \Delta V(r, Q)=\sum_{t} \frac{\partial V}{\partial Q_{t}} Q_{t}+\frac{1}{2} \sum_{t t^{\prime}} \frac{\partial^{2} V}{\partial Q_{t} \partial Q_{t^{\prime}}} Q_{t} Q_{t^{\prime}},
\end{aligned}
$$

and the crude adiabatic wave function in the form

$$
\psi_{n}(r, Q)=\phi_{0}(r, 0) \chi_{n}(Q),
$$

where $\phi_{0}$ and $\chi_{n}$ are the electronic and vibrational parts, respectively. We write the electronic part of the function in Eq. (6) with a 0 as one argument to emphasize that it is independent of $Q_{t}$ and a solution of the electronic Hamiltonian for the $Q_{t}=0$ nuclear geometry, which is at the minimum of the nuclear potential. Since the normal coordinates are arranged to diagonalize the vibrational potential we therefore have

$$
\left\langle\phi_{0}(r, 0)|\Delta V(r, Q)| \phi_{0}(r, 0)\right\rangle=\frac{1}{2} \sum_{t} f_{t} Q_{t}^{2},
$$

where $f_{t}$ are the harmonic force constants for the vibrational motion of the neutral molecule.

We construct the asymptotic continuum functions by combining $\psi(r, Q)$ with a single particle continuum function using the appropriate antisymmetrizer,

$$
\Psi_{n}(E, r, Q)=A\left[\phi_{0}(r, 0) \epsilon(E, r)\right] \chi_{n}(Q) .
$$

The Hamiltonian now refers to one more electron:

$$
\begin{aligned}
H= & T(r)+T(Q)+V(r, 0)+\Delta V(r, Q)+T\left(r_{\epsilon}\right) \\
& +V_{\text {int }}\left(r_{\epsilon}, Q\right),
\end{aligned}
$$

and with this we obtain

$$
\begin{aligned}
& {\left[H_{a a}\left(E^{\prime}, E\right)\right]_{n^{\prime} n}} \\
& \quad=\left\langle\Psi_{n^{\prime}}\left(E^{\prime}\right)|H| \Psi_{n}(E)\right\rangle \\
& \quad=\left(E+E_{n}^{(\mathrm{vib})}\right) \delta\left(E^{\prime}-E\right) \delta_{n^{\prime} n}+V_{n^{\prime} n}^{(\mathrm{int})}\left(E^{\prime}, E\right) .
\end{aligned}
$$

The resonant portion of our basis will consist of one or more, possibly degenerate, quasibound state functions. These represent the neutral molecule with one extra electron, i.e., these are the wave functions for the temporary negative ion, a term referring to the system in the resonance state. We note the further point that the electronic part of the resonant function is for a molecular geometry identical with that of the neutral target molecule. We write the function as

$$
\dot{\Psi_{i p}^{(b)}}(r, Q)=\phi_{i}^{(b)}(r, 0) \chi_{p}(Q),
$$


where the vibrational factors are the same as those in Eq. (6). Again, this is the crude adiabatic prescription. The corresponding block of the Hamiltonian is then

$$
\begin{aligned}
\left(H_{b b}\right)_{i^{\prime} p^{\prime} i p}= & {\left[E_{i}^{(b)}+E_{p}^{(\mathrm{vib})}\right] \delta_{i^{\prime} i} \delta_{p^{\prime} p}+\sum_{t}\left\langle i^{\prime}\left|\frac{\partial V}{\partial Q_{t}}\right| i\right\rangle } \\
& \times\left\langle p^{\prime}\left|Q_{t}\right| p\right\rangle+\frac{1}{2} \sum_{t t^{\prime}}\left[\left\langle i^{\prime}\left|\frac{\partial^{2} V}{\partial Q_{t} \partial Q_{t^{\prime}}}\right| i\right\rangle\right. \\
& \left.-f \delta_{t t^{\prime}} \delta_{i^{\prime} i}\right]\left\langle p^{\prime}\left|Q_{t} Q_{t^{\prime}}\right| p\right\rangle,
\end{aligned}
$$

where the vibrational energy term $E_{p}^{(\mathrm{vib})}$ is the same as that in Eq. (10). Nonzero values of the matrix elements of the partial derivatives in the second and third lines of Eq. (12) reflect the different forces on the nuclei in $b$ states from those in $a$ states.

Finally, we have for the $H_{a b}$ block of the Hamiltonian

$$
\begin{aligned}
{\left[H_{a b}(E)\right]_{n ; i p}=} & V_{n ; i p}(E) \\
= & \delta_{n p} \gamma_{i}^{(b)}(E)+\sum_{t}\left\langle\phi_{0} \epsilon_{E}\left|\frac{\partial V}{\partial Q_{t}}\right| i\right\rangle\left\langle n\left|Q_{t}\right| p\right\rangle \\
& +\frac{1}{2} \sum_{t t^{\prime}}\left\langle\phi_{0} \epsilon_{E}\left|\frac{\partial^{2} V}{\partial Q_{t} \partial Q_{t^{\prime}}}\right| i\right\rangle\left\langle n\left|Q_{t} Q_{t^{\prime}}\right| p\right\rangle
\end{aligned}
$$

The basis functions are assumed to satisfy the various orthogonalities and normalizations given by

$$
\begin{aligned}
& \left\langle\Psi_{n^{\prime}}\left(E^{\prime}\right) \mid \Psi_{n}(E)\right\rangle=\delta_{n^{\prime} n} \delta\left(E^{\prime}-E\right), \\
& \left\langle\Psi_{i^{\prime} p^{\prime}}^{(b)} \mid \Psi_{i p}^{(b)}\right\rangle=\delta_{i^{\prime} i} \delta_{p^{\prime} p}, \\
& \left\langle\Psi_{n}(E) \mid \Psi_{i p}^{(b)}\right\rangle=0 .
\end{aligned}
$$

It will be observed that each of the blocks of the $H$ matrix is the sum of two terms, the first of which is diagonal and the second not. In Eq. (10), the continuumcontinuum block, we will ignore the second term. This contributes to nonresonant potential scattering excitation of vibrations and is not expected to be large. Estrada et al. ${ }^{20}$ neglect the same term in their treatment.

In Eq. (12), the resonant-resonant block, we see that the VC produces part of the off-diagonal matrix elements, but some are introduced through using the vibrational functions of the neutral ground state. Let us note the circumstances under which the off-diagonal terms in Eq. (12) might vanish. This would correspond to a hypothetical situation in which the extra electron in the resonant function does not change the nuclear potential from its form in the neutral molecule. A specific example might be an electron in an orbital that is neither bonding nor antibonding but truly nonbonding. Consequently, the normal coordinates are correct for the negative ion state as well as for the neutral molecule and the first derivative terms would vanish. The second derivative matrix elements are diagonal with respect to $i$ and $i^{\prime}$ with the diagonal elements just $f_{i}$. Therefore, the second derivative terms also would vanish. Having seen how this might happen, we conclude that a resonant state with so minor an effect on the molecule as this does not merit being treated as a resonance.

In Eq. (13), the resonant-continuum block, the VC terms are not expected to be zero, in general.

\section{A PERTURBATION EXPRESSION FOR $T_{a a}$}

We now write the resonant-continuum and resonantresonant blocks of the Hamiltonian as

$$
\begin{aligned}
& H_{b b}=E^{(b)}+\Delta B, \\
& H_{a b}=\gamma^{(b)}+\Delta V,
\end{aligned}
$$

and this produces a similar expression for $F(E)$ :

$$
\begin{aligned}
F_{b b}(E) & =f(E)+\Delta F(E), \\
f(E)= & \int \frac{\bar{\gamma}^{(b)} \gamma^{(b)} d E^{\prime}}{E-E^{\prime}}+i \pi \bar{\gamma}^{(b)} \gamma^{(b)}, \\
\Delta F(E)= & \int \frac{\left(\Delta \bar{V} \gamma^{(b)}+\bar{\gamma}^{(b)} \Delta V+\Delta \bar{V} \Delta V\right) d E^{\prime}}{E-E^{\prime}} . \\
& +i \pi\left(\Delta \bar{V} \gamma^{(b)}+\bar{\gamma}^{(b)} \Delta V+\Delta \bar{V} \Delta V\right),
\end{aligned}
$$

where the bar over a symbol indicates the Hermitian conjugate. In Eq. (15) and the first line of Eq. (16) the first term is diagonal with respect to vibrational quantum numbers, but the second is not. When Eqs. (15) and (16) are substituted into Eq. (4), we obtain

$$
\begin{aligned}
T_{a a}= & \left(\gamma^{(b)}+\Delta V\right)\left[E-E^{(b)}-f(E)-\Delta B\right. \\
& -\Delta F(E)]^{-1}\left(\bar{\gamma}^{(b)}+\Delta \bar{V}\right), \\
= & \gamma^{(b)}\left[E-E^{(b)}-f(E)\right]^{-1} \bar{\gamma}^{(b)}+\Delta V\left[E-E^{(b)}\right. \\
& -f(E)]^{-1} \bar{\gamma}^{(b)}+\text { c.t. }+\gamma^{(b)}\left[E-E^{(b)}\right. \\
& -f(E)]^{-1}[\Delta B+\Delta F(E)]\left[E-E^{(b)}\right. \\
& -f(E)]^{-1} \bar{\gamma}^{(b)}+\cdots,
\end{aligned}
$$

where the bar again indicates the Hermitian conjugate. In the second equation of Eqs. (17) the term on the first line is diagonal in the vibrational quantum numbers and does not lead to vibrational excitation. On the other hand, this is not true of $\Delta B, \Delta V$, and $\Delta F(E)$, and they do contribute to excitation.

Although the expansion in Eqs. (17) is not expected to converge rapidly enough (at least at all energies) to be useful for numerical calculations, it does show how the various asymptotic statcs arc connected through excited virtual states (in the perturbation sense) and, hence, provides us the symmetry selection rules we require.

We see that there are two independent ways that VC can produce nonzero elements in the transition matrix that lead to inelastic vibrational excitation. The first of these, represented in Eq. (15) by $\Delta B$, we call internal vibronic coupling. It operates within and between the quasibound resonant states that we have. The interactions here lead to a number of phenomena with different names such as the 
Jahn-Teller or the Renner-Teller effects. When one has two different arbitrary electronic states the generic term, VC, is usually applied. ${ }^{15}$

The second, represented in Eq. (15) by $\Delta V$, we call external vibronic coupling. It operates between the resonant states and the asymptotic continuum states. In addition, it appears in two different places in the perturbation expression (17), directly as $\Delta V$, itself, and in the morc complicated expression, $\Delta F(E)$. In our treatment the effect of $\Delta B$ and $\Delta F(E)$ together represent a matrix version of the complex, nonlocal potential discussed by Estrada et al. ${ }^{12}$

\section{SYMMETRY ANALYSIS OF VIBRATIONAL EXCITATIONS IN BENZENE}

We shall show the application of these relations by making a complete analysis for benzene of the symmetry requirements for vibrational excitation by electron impact at resonant energies. Wong and Schulz ${ }^{1}$ have studied this experimentally, determining vibrational excitation functions and their angular distributions. Benzene is an ideal substance for this illustration, sincc it provides, within one molecule, almost all of the phenomena that we expect to be important. We note, in particular, that benzene has:

(a) a shape resonance near $1.1 \mathrm{eV}$ with a state symmetry ${ }^{2} E_{2 u}{ }^{21}$ It is doubly degenerate and shows with the appropriate vibrational modes the typical behavior associated with the Jahn-Teller effect;

(b) a shape resonance near $4.8 \mathrm{eV}$ with a state symmetry of ${ }^{2} B_{2 g}{ }^{21}$ This is nondegenerate and shows behavior expected with such statcs;

(c) a very broad feature centered near $8.0 \mathrm{eV} .{ }^{22,23}$ This is probably a shape resonance associated with $\mathrm{C}-\mathrm{C}$ and $\mathrm{C}-\mathrm{H} \sigma^{*}$ orbitals in the molecule. This latter resonance is so broad that it appears to overlap with the ${ }^{2} B_{2 g}$ resonance. These two are thus expected to provide an example of the type of vibronic interaction between resonances discussed by Estrada et al. ${ }^{12}$

The numbering of the normal vibrations in benzene will be important in detailing the analysis. Unfortunately, there is not unanimity on this in the literature. We follow the scheme used by Herzberg, ${ }^{24}$ which is repeated in Table II, for convenience. Table II also gives the vibrational energies, and indicates those vibrational modes associated principally with $\mathrm{C}-\mathrm{C}$ and $\mathrm{C}-\mathrm{H}$ bond stretching motions.

\section{A. The ${ }^{2} E_{2 u}$ resonance at $1.1 \mathrm{eV}$}

Internal VC within this doubly degenerate electronic state is the sort associated with the Jahn-Teller effect, ${ }^{15}$ i.e., the term $\Delta V(r, Q)$ in Eq. (5) produces an interaction between vibrational and electronic motions that is exceptionally large because of the electronic state energy degeneracy in zero order. ${ }^{25}$ The effect of the symmetry of the electronic state is given by the symmetrized square, $\left[e_{2 u}^{2}\right]$. The analysis appears in the Appendix. Vibrational modes with symmetries matching the symmetrized square will interact in the first order term of $\Delta V(r, Q)$, and the results are shown in Table III.

So far as symmetry is concerned we expect vibrations of type $v_{1}, v_{2}\left(a_{1 g}\right)$ and $v_{15}, v_{16}, v_{17}, v_{18}\left(e_{2 g}\right)$ to couple com-
TABLE II. Symmetry species and vibrational energies of benzene. This is the scheme used by Herzberg (Ref. 24).

\begin{tabular}{cccl}
\hline \hline $\begin{array}{c}\text { Symmetry } \\
\text { species }\end{array}$ & Numbering & $\omega$ & \multicolumn{1}{c}{ Motion } \\
\hline$a_{1 g}$ & $v_{1}$ & $380 \mathrm{meV}$ & $\mathrm{C}-\mathrm{H}$ str \\
& $v_{2}$ & 123 & $\mathrm{C}-\mathrm{C}$ str \\
$a_{2 g}$ & $v_{3}$ & $148^{\mathrm{a}}$ & $\mathrm{C}-\mathrm{H} \|$ bend \\
$a_{2 u}$ & $v_{4}$ & 83 & $\mathrm{C}-\mathrm{H} \perp$ bend \\
$b_{1 u}$ & $v_{5}$ & 380 & $\mathrm{C}-\mathrm{H}$ str \\
& $v_{6}$ & 125 & $\mathrm{C}-\mathrm{C}-\mathrm{C} \|$ bend \\
$b_{2 g}$ & $v_{7}$ & $188^{\mathrm{a}}$ & $\mathrm{C}-\mathrm{H} \perp$ bend \\
& $v_{8}$ & $67^{\mathrm{a}}$ & $\mathrm{C}-\mathrm{C}-\mathrm{C} \perp$ bend \\
$b_{2 u}$ & $v_{9}$ & $230^{\mathrm{a}}$ & $\mathrm{C}-\mathrm{C}$ str \\
& $v_{10}$ & $142^{\mathrm{a}}$ & $\mathrm{C}-\mathrm{H} \|$ bend \\
$e_{1 g}$ & $v_{11}$ & 105 & $\mathrm{C}-\mathrm{H} \perp$ bend \\
$e_{1 u}$ & $v_{12}$ & 384 & $\mathrm{C}-\mathrm{H}$ str \\
& $v_{13}$ & 184 & $\mathrm{C}-\mathrm{C}$ str \\
$e_{2 g}$ & $v_{14}$ & 129 & $\mathrm{C}-\mathrm{H} \|$ bend \\
& $v_{15}$ & 376 & $\mathrm{C}-\mathrm{H}$ str \\
& $v_{16}$ & 196 & $\mathrm{C}-\mathrm{C}$ str \\
& $v_{17}$ & 146 & $\mathrm{C}-\mathrm{H} \|$ bend \\
$e_{2 u}$ & $v_{18}$ & 75 & $\mathrm{C}-\mathrm{C}-\mathrm{C} \|$ bend \\
& $v_{19}$ & $144^{\mathrm{a}}$ & $\mathrm{C}-\mathrm{H} \perp$ bend \\
\hline \hline
\end{tabular}

${ }^{\mathrm{a} C a l c u l a t e d .}$

ponents of the ${ }^{2} E_{2 u}$ resonance and hence to appear in vibrational excitation. Not all of these appear; certain dynamical principles also apply. It is well known that orbitals temporarily occupied when observing shape resonances in molecules like benzene are antibonding. Simple theories of bonding tell us that the ${ }^{2} E_{2 u}$ state in benzene is antibonding with respect to the $\mathrm{C}-\mathrm{C}$ bonds but not with respect to the $\mathrm{C}-\mathrm{H}$ bonds. $v_{2}\left(a_{1 g}\right)$ and $v_{16}\left(e_{2 g}\right)$ are the two vibrational modes associated with $\mathrm{C}-\mathrm{C}$ stretching motions and these are just the modes seen in vibrational excitation in benzene. This has already been noted by Wong and Schulz. ${ }^{1}$

The vibrational modes that can be excited through the second order terms in $\Delta V(r, Q)$ are shown in Table IV. If we again assume involvement of only $\mathrm{C}-\mathrm{C}$ stretches we expect only $2 v_{2}\left(a_{1 g}\right), 2 v_{16}\left(e_{2 g}\right)$, and $v_{2}+v_{16}$ excitations, which seen as parts of series. Such excitations will also be produced by higher order terms in Eq. (17), and we therefore cannot ascribe their appearance to a specific mechanism.

The form of Eq. (17) indicates that the scattered electron for these excitations will have $l$ values appropriate for the $e_{2 u}$ symmetry. Table $\mathrm{V}$ gives the symmetry analysis of the spherical harmonics for irreducible representations (IR) of $D_{6 h}$, and one sees that $l=3$ is the leading value for

TABLE III. Vibrational modes in benzene excited by internal VC and first order derivatives.

\begin{tabular}{ccccccc}
\hline \hline $\begin{array}{c}\text { Resonance } \\
\text { state }\end{array}$ & $\begin{array}{c}\text { Quassibound } \\
\text { state symm. }\end{array}$ & $\begin{array}{c}\text { Symmetrized } \\
\text { square }\end{array}$ & \multicolumn{4}{c}{$\begin{array}{c}\text { Possible } \\
\text { modes }\end{array}$} \\
\hline${ }^{2} E_{2 u}$ & $e_{2 u}$ & $a_{1 g}$ & $v_{1}$ & $v_{2}$ & & \\
& & $e_{2 g}$ & $v_{15}$ & $v_{16}$ & $v_{17}$ & $v_{18}$ \\
${ }^{2} B_{2 g}$ & $b_{2 g}$ & $a_{1 g}$ & $v_{1}$ & $v_{2}$ & & \\
\hline \hline
\end{tabular}


TABLE IV. Vibrational modes in benzene excited by internal VC and second order derivatives.

\begin{tabular}{|c|c|c|c|}
\hline $\begin{array}{l}\text { Resonance } \\
\text { state }\end{array}$ & $\begin{array}{l}\text { Quasibound } \\
\text { state symm. }\end{array}$ & $\begin{array}{l}\text { Symmetrized } \\
\text { square }\end{array}$ & $\begin{array}{l}\text { Possible } \\
\text { modes }\end{array}$ \\
\hline${ }^{2} E_{2 u}$ & $e_{2 u}$ & $e_{2 g}$ & $\begin{array}{l}2 v_{i}, i=1,2, \ldots, 20 \\
v_{i}+v_{j}, i \neq j, i, j=12,13,14 \\
v_{i}+v_{j}, i \neq j, i, j=15,16,17,18 \\
v_{i}+v_{j}, i \neq j, i, j=19,20 \\
2 v_{i}, i=11,12, \ldots, 20 \\
v_{i}+v_{j}, i \neq j, i, j=12,13,14 \\
v_{i}+v_{j}, i \neq j, i, j=15,16,17,18 \\
v_{i}+v_{j}, i \neq j, i, j=19,20 \\
v_{i}+v_{j}, i=1,2,3 ; j=15,16,17,18 \\
v_{i}+v_{j}, i=7,8 ; j=11 \\
v_{i}+v_{j}, i=5,6,9,10 ; j=12,13,14 \\
v_{i}+v_{j}, i=4 ; j=19,20\end{array}$ \\
\hline${ }^{2} B_{2 g}$ & $b_{2 g}$ & $a_{1 g}$ & $\begin{array}{l}2 v_{i}, i=1,2, \ldots, 20 \\
v_{i}+v_{j}, i \neq j, i, j=12,13,14 \\
v_{i}+v_{j}, i \neq j, i, j=15,16,17,18 \\
v_{i}+v_{j}, i \neq j, i, j=19,20\end{array}$ \\
\hline
\end{tabular}

$e_{2 u}$. This tells us that the angular distribution of the scattering for excitation functions associated with these modes should be predominately that due to an $f$ wave, ${ }^{26}$ which is observed.

External VC for the ${ }^{2} E_{2 u}$ resonance offers more possibilities since the continuum functions, in principle, support all symmetry species. Neverthcless, as we argued in I, continuum functions with very high $l$ values are so small in the vicinity of the molecule that integrals like $V_{n ; i p}$ in Eq. (13) will be negligible in such cases. In Table VI we give the vibrational modes possibly excited by the first order terms in $\Delta V(r, Q)$ for $l=0,1$, and 2 . If, because of the zero angular momentum barrier, we assume that the $s$ wave will predominate, the $v_{19}, v_{20}\left(e_{2 u}\right)$ modes should be excited. $v_{20}\left(e_{2 u}\right)$ is the carbon ring puckering motion, and the quasibound state orbital $e_{2 u}$ is located principally on the carbon atoms so this is the mode we expect to be excited by this mechanism. $v_{20}\left(e_{2 u}\right)$ does appear in the experimental results, and it appears with an angular distribution indicating $s$-wave involvement. ${ }^{1}$

TABLE V. Frequencies of $D_{6 h}$ IRs in the spherical harmonics for $l$ from 0 to 12 .

\begin{tabular}{|c|c|c|c|c|c|c|c|c|c|c|c|c|c|}
\hline \multirow{2}{*}{$\begin{array}{l}\text { Symmetry } \\
\text { species }\end{array}$} & \multicolumn{13}{|c|}{$l$} \\
\hline & 0 & 1 & 2 & 3 & 4 & 5 & 6 & 7 & 8 & 9. & 10 & 11 & 12 \\
\hline$a_{1 g}$ & 1 & & 1 & & 1 & & 2 & & 2 & & 2 & & 3 \\
\hline$a_{1 u}$ & & & & & & - & & 1 & & 1 & & 1 & \\
\hline$a_{2 g}$ & & & & & & & 1 & & 1 & & 1 & & 2 \\
\hline$a_{2 u}$ & & 1 & & 1 & & 1 & & 2 & & 2 & & 2 & \\
\hline$b_{1 g}$ & & & & & 1 & & 1 & & 1 & & 2 & & 2 \\
\hline$b_{1 u}$ & & & & 1 & & 1 & & 1 & & 2 & & 2 & \\
\hline$b_{2 g}$ & & & & & 1 & & 1 & & 1 & & 2 & & 2 \\
\hline$b_{2 u}$ & & & & 1 & & 1 & & 1 & & 2 & & 2 & \\
\hline$e_{1 g}$ & & & 1 & & 1 & & 2 & & 3 & & 3 & & 4 \\
\hline$e_{1 u}$ & & 1 & & 1 & & 2 & & 3 & & 3 & & 4 & \\
\hline$e_{2 g}$ & & & 1 & & 2 & & 2 & & 3 & & 4 & & 4 \\
\hline$e_{2 u}$ & & & & 1 & & 2 & & 2 & & 3 & & 4 & \\
\hline
\end{tabular}

TABLE VI. Vibrational modes in benzene excited by external VC and first derivatives for $l=0,1$, and 2 .

\begin{tabular}{ccccccc}
\hline $\begin{array}{c}\text { Resonance } \\
\text { state }\end{array}$ & $\begin{array}{c}\text { Continuum } \\
l \text { value }\end{array}$ & $\begin{array}{c}\text { Combined } \\
\text { states }\end{array}$ & \multicolumn{4}{c}{$\begin{array}{c}\text { Possible } \\
\text { modes }\end{array}$} \\
\hline${ }^{2} E_{2 u}$ & $s$ & $e_{2 u}$ & $v_{19}$ & $v_{20}$ & & \\
& $p$ & $e_{2 g}$ & $v_{15}$ & $v_{16}$ & $v_{17}$ & $v_{18}$ \\
& & $b_{1 g}$ & none & & & \\
& & $b_{2 g}$ & $v_{7}$ & $v_{8}$ & & \\
& $d$ & $e_{1 g}$ & $v_{11}$ & & & \\
& & $2 e_{2 u}$ & $v_{19}$ & $v_{20}$ & & \\
& & $b_{1 u}$ & $v_{5}$ & $v_{6}$ & & \\
& & $b_{2 u}$ & $v_{9}$ & $v_{10}$ & & \\
& & $e_{1 u}$ & $v_{12}$ & $v_{13}$ & $v_{14}$ & \\
${ }^{2} B_{2 g}$ & & $a_{1 u}$ & none & & & \\
& & $a_{2 u}$ & $v_{4}$ & & & \\
& & $b_{2 g}$ & $v_{7}$ & $v_{8}$ & & \\
& & $b_{2 u}$ & $v_{9}$ & $v_{10}$ & & \\
& $d$ & $e_{2 u}$ & $v_{19}$ & $v_{20}$ & & \\
& & $b_{2 g}$ & $v_{7}$ & $v_{8}$ & & \\
& & $e_{1 g}$ & $v_{11}$ & & & \\
\hline \hline
\end{tabular}

Table VIII gives the possible modes excited for external VC and the second order terms in $\Delta V(r, Q)$ and continuum functions for $l=0,1$, and 2 . Considering only $s$ waves again, we expect $v_{2}+v_{20}$ and $v_{16}+v_{20}$. These combinations are expected because they involve $\mathrm{C}-\mathrm{C}$ stretch and ring puckering at the same time. An $s$-wave angular distribution is expected. The first and second order excitations will again be augmented by higher order terms in Eq. (17) so series based upon these modes may be strong enough to see.

\section{B. The ${ }^{2} B_{2 g}$ resonance at $4.8 \mathrm{eV}$}

The internal VC involving first derivatives is shown in Table III and that with second derivatives in Table IV. For this nondegenerate state $v_{1}, v_{2}\left(a_{1 g}\right)$, any $2 v_{i}$, and several pairs of degenerate $v_{i}+v_{j}$ combinations are possible. The only one of these that is seen strongly is the $v_{1}\left(a_{1 g}\right)$ sequence. Although one might again expect $v_{2}\left(a_{1 g}\right)$ because of the $\mathrm{C}-\mathrm{C}$ antibonding nature of the $b_{2 g}$ orbital, this does not appear. Detailed $a b$ initio calculations would be required to see why this occurs. Table $V$ shows that $l=4$ is the leading term in an orbital of $b_{2 g}$ symmetry, and an angular distribution consistent with $g$ waves is seen.

The external VC with first order terms involving continuum $s$ waves is expected to excite $v_{7}, v_{8}\left(b_{2 g}\right)$ modes; $v_{7}\left(b_{2 g}\right)$ is seen experimentally. The angular distribution is consistent with an $s$ wave.

Table VII shows that several $v_{i}+v_{j}$ combinations are possible from second order terms, but these are apparently too faint to be seen experimentally.

\section{Interaction of the ${ }^{2} B_{2 g}$ resonance at $4.8 \mathrm{eV}$ with higher resonances}

A recent article by Ben Arfa and Tronc ${ }^{13}$ gives a symmetry analysis of vibrational excitation in benzene that invokes vibronic interactions between two resonances, bas- 
TABLE VII. Vibrational modes in benzene excited by external VC and second order derivatives for $l=0,1$, and 2 .

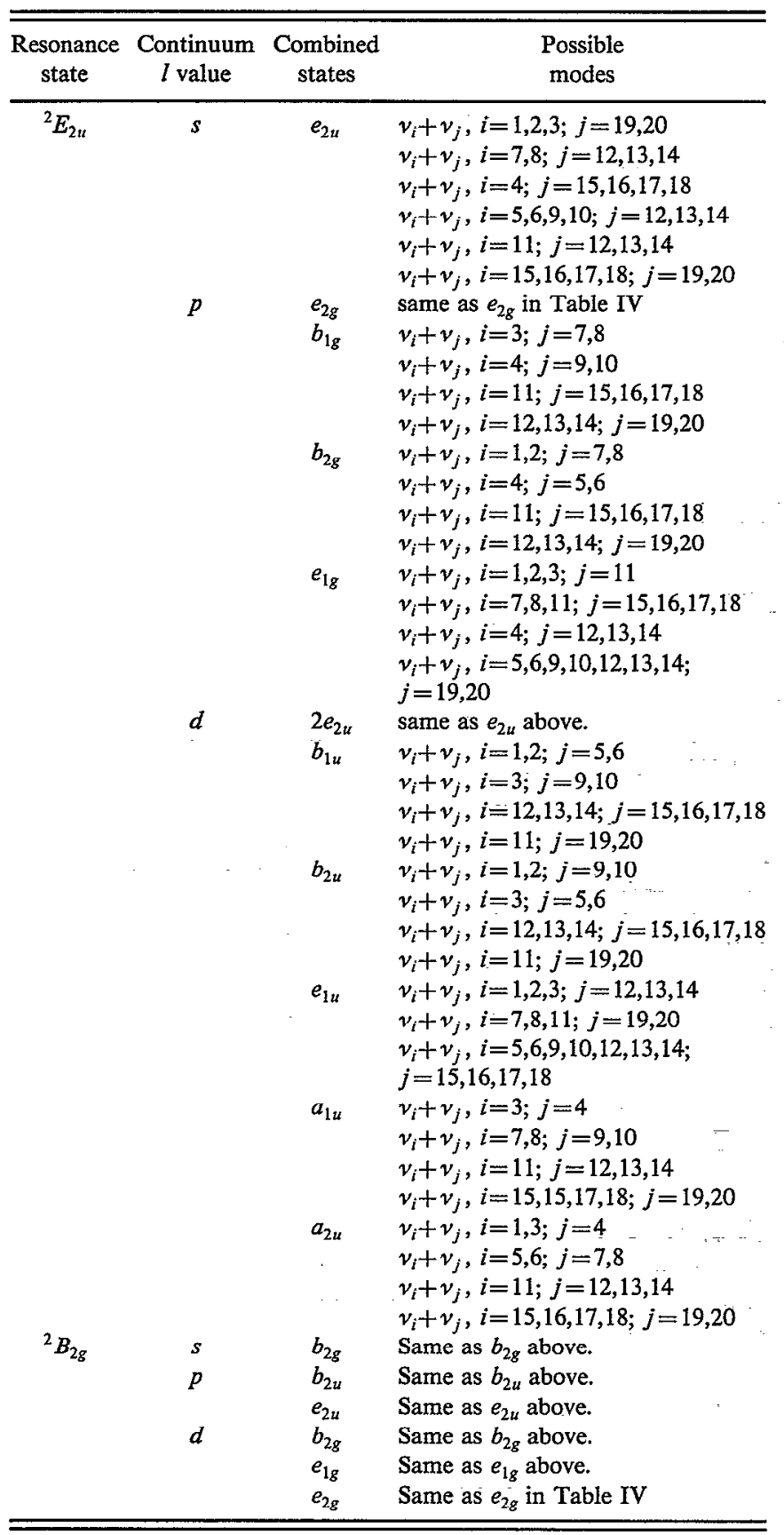

ing their discussion on the treatment of this by Estrada et al. ${ }^{12}$ We shall examine this proposal to see how it fits into the scheme outlined in this article.

From simple bonding theory the higher resonance or resonances are expected to be associated with $\mathrm{C}-\mathrm{C}$ and $\mathrm{C}-\mathrm{H} \sigma^{*}$ bonds in benzene. Table VIII gives the symmetry species that such orbitals support, and it is seen that the two types support nearly the same IRs. An $a_{1 g}$ orbital consists of only $\mathrm{C}-\mathrm{H} \sigma^{*}$ bond orbitals and an $a_{2 g}$ orbital of only $\mathrm{C}-\mathrm{C} \sigma^{*}$ bond orbitals. In the $b_{1 u}, e_{1 u}$, and $e_{2 g}$ states the two bond types will be mixed. By examining the number of nodes in the orbitals we expect the $a_{1 g}$ to be the lowest in energy followed by the $e_{1 u}$ degenerate pair, and
TABLE VIII. Symmetry functions of $\mathrm{C}-\mathrm{C}$ and $\mathrm{C}-\mathrm{H}$ antibonding orbitals in benzene.

\begin{tabular}{cc}
\hline Bond type & Species supported \\
\hline $\mathrm{C}-\mathrm{H} \sigma^{*}$ & $a_{1 g}+b_{1 u}+e_{1 u}+e_{2 g}$ \\
$\mathrm{C}-\mathrm{C} \sigma^{*}$ & $a_{2 g}+b_{1 u}+e_{1 u}+e_{2 g}$ \\
\hline
\end{tabular}

we restrict our attention to this pair of symmetries. From Table $\mathrm{V}$ we see that $e_{1 u}$ has a leading $l=1$, and we can hypothesize that this is a principal contributor to the visible resonance, since it is normally supposed that an angular momentum barrier is necessary for shape resonances. (In molecules as large as benzene the possibility that high energy' resonances contain considerable admixtures of core excited states has been suggested, but this is virtually unexplored theoretically.) The $a_{1 g}$ has a leading $l=0$.

Internal VC can operate between the ${ }^{2} B_{2 g}$ state and the ${ }^{2} A_{1 g}$ or the ${ }^{2} E_{1 u}$ states based upon the $\sigma^{*}$ orbitals. As far as symmetry is concerned, the allowed vibrational excitations are identical to the allowed vibrational excitations from external VC that the ${ }^{2} B_{2 g}$ state has with the $s$ or the $m= \pm 1$ components of the $p$ type continuum functions.

The relationship between these two mechanisms is shown, in the opinion of the author, most clearly by Fano's configuration interaction treatment of quasibound states with the continuum. ${ }^{17}$ In this approach one writes the solution to the scattering problem as a linear combination of the quasi bound state and the continuum [see Fano's Eq. (2)]. In our notation this is

$$
\sum_{i p} a_{i p}(E) \Psi_{i p}^{(b)}+\sum_{n} \int b_{n}\left(E, E^{\prime}\right) \Psi_{n}\left(E^{\prime}\right) d E^{\prime},
$$

where $a$ and $b$ are the expansion coefficients. From this viewpoint, the internal vibronic interaction between two different resonances and the external vibronic interaction between one of the resonances and the continuum are two aspects of the same interaction. Specifically, in benzene, we may expect that the $\mathrm{C}-\mathrm{H} \sigma^{*} a_{1 g}$ quasibound state will be mixed to some extent with the $s$-wave continuum function according to Eq. (18). The amount of this mixing will be energy dependent and appears to be available only from theoretical considerations. Therefore, the interaction represented by Fq. (18) might enhance the amplitude of the $s$-wave continuum function in the vicinity of the molecule and make the value of the corresponding $\Delta V$ term in Eq. (17) larger than it would be in the absence of the $\sigma^{*}$ function. But either way, the result is an angular distribution with $s$-wave character. The amount of this enhancement would seem to require $a b$ initio calculations to determine.

In a similar manner, the $e_{1 u}$ resonance could enhance the amplitude of some components of the $p$-wave continuum function near the molecule and increase the amount of $p$-wave character in the angular distribution. Again, detailed calculations appear necessary to assess the size of the effect in a specific case. 


\section{DISCUSSION}

At the time Wong and Schulz ${ }^{1}$ made their measurements on benzene, the appearance of $s$-type angular distributions for some of the vibrational excitation functions from resonances with as high leading $l$ values as ${ }^{2} E_{2 u}$ and ${ }^{2} B_{2 g}$ have was surprising. In I the author coined the phrase "s-wave leakage" to describe the possibility that nuclear distortions can essentially transform the high $l$ values into 0 . In most ways the present treatment duplicates the results of $I$. The principal difference is that we have here included the interaction between two quasibound states in order to compare more directly with Estrada et al. ${ }^{12}$

This latter treatment does not distinguish the two terms $\Delta B$ and $\Delta F(E)$ in Eq. (17) the way ours does. In fact, they do not use the crude adiabatic basis, but what is called (in spite of their terminology) the Born-Huang adiabatic basis. ${ }^{15}$ This produces vibronic interactions in a completely equivalent but apparently quite different way. One of the principal differences is the explicit appearance in the Hamiltonian of momentum operators associated with the $Q$ 's. It should be emphasized that such quantities appear nowhere in our treatment, an absence characteristic of the crude adiabatic basis.

Estrada et al. capped their treatment with a model calculation of vibronic interaction within a complex local potential that showed interesting angular distribution effects. In their model the coupling is quite strong and the states were not very similar in symmetry structure to those in benzene. In any event they did not attempt to analyze their results in any way similar to the present division of effects into internal and external VC.

As stated in Sec. I, our goal here was to show how a symmetry analysis of the expected vibrational excitations could be made. This is most simply done with the crude adiabatic basis and leads to the separation of effects we have discussed.

Other molecules can be analyzed using the same principles, but one should note one difference between the discussion here and how the analysis would proceed for a linear molecule. The so-called linear Jahn-Teller effect does not operate in linear molecules but is replaced by the Renner-Teller effect. ${ }^{15}$ Only even quantum number states of bending mode vibrations can be excited in this way. The odd quantum number states of these modes can be excited only by external VC or VC with other resonances of appropriate symmetries.

\section{CONCLUSION}

In conclusion we state the symmetry rules we have discussed, for simplicity, giving only those based upon the first order terms in $\Delta V(r, Q)$ (a) and (b) operate independently, but in some cases may result in overlapping symmetry types.

(a) For a shape resonance with the spatial symmetry $\Gamma$, one calculates the symmetrized square representation $\left[\Gamma^{2}\right]$. Vibrations of the same symmetries as those contained in $\left[\Gamma^{2}\right]$ can be excited. When more than one vibration exists of a possible type, dynamical consider- ations may cause many of them to appear with insufficient intensity to observe. The angular distributions of these excitation functions are determined principally by the leading $l$ value in $\Gamma$.

(b) For a shape resonance with the spatial symmetry $\Gamma$, we expect vibrations of the same symmetry, $\Gamma$, to appear. Again, if more than one vibration of this type occurs, dynamical considerations will determine the relative intensities. In this case the excitation function should show a nearly $s$-wave type angular distribution.

As we have seen, second order effects occur. These are much more complicated and rules are difficult to codify. Measurements of more substances will be required to decide on the relative importance of these terms. The measurements in benzene indicate that the first order rules predict the principal peaks in the resonant energy loss spectrum and the shapes of the angular distributions, but some series are seen.

\section{APPENDIX: SYMMETRY ANALYSIS OF MATRIX ELEMENTS OF THE ELECTRON-NUCLEAR POTENTIAL}

An analysis of the symmetry of matrix elements for polyatomic molecules are most simply carried out in a molecular framework. If we were interested in angular distributions of gaseous molecules, the molecular framework result must be transformed into a laboratory framework. But, if we are merely interested in what can or cannot happen, we may remain in the molecular framework.

For a molecule with $N$ atoms, $n$ electrons, and $M$ $(=3 N-6)$ vibrational normal coordinates, the electronnucleus potential may be written

$$
V=V\left(r_{1} \cdots r_{n}, Q_{1} \cdots Q_{M}\right)=V\left(r_{i}, Q_{j}\right),
$$

where $r_{i}$ is the vector position of the $i$ th electron and $Q_{j}$ is the $j$ th normal coordinate. As we indicated, the $r$ vectors give the position of the electrons in a molecule fixed coordinate system. The vector position of the $\alpha$ th nucleus, $\rho_{\alpha}$, is a linear function of the $Q_{j}{ }^{27}$ The nuclei are all in their equilibrium positions when all the $Q$ 's are zero.

The symmetry point group of the molecule is the set of proper and improper rotations that carries the equilibrium positions of the nuclei into themselves. Let $R$ be an element of the group. The crucial property of the potential function is

$$
R V=V\left(R^{-1} r_{i}, R^{-1} Q_{j}\right)=V,
$$

i.e., it is invariant when subjected to the group operations. Well known methods ${ }^{27}$ allow one to determine the complete representation of the symmetry group supported by the normal vibrations; we denote this by the symbol $\Gamma_{Q}$. In addition, the normal coordinates belong to various irreducible representations (IR) of the symmetry group, and a complete specification of the properties requires three labels. Thus when we write $Q_{n \Gamma j}, j=1,2, \ldots, g_{\Gamma}$, it indicates the $n$th occurrence of the $\Gamma$ IR of degeneracy $g_{\Gamma}$. We shall use this detailed notation only when needed.

According to Eq. (5) we represent $V$ as a Taylor's expansion in the $Q$ 's 


$$
V=V\left(r_{i}, 0\right)+\sum_{j} \frac{\partial V}{\partial Q_{j}} Q_{j}+\frac{1}{2} \sum_{j, k} \frac{\partial^{2} V}{\partial Q_{j} \partial Q_{k}} Q_{j} Q_{k}+\cdots,
$$

where each of the partial derivatives is a function of the $r \mathrm{~s}$. Because of the linear independence of the $Q$ 's and the invariance represented by Eq. (A2), each of the individual terms in Eq. (A3) is also invariant under transformation by the group element, $R$. This has consequences for the transformation properties of the partial derivatives. Taking the linear terms as an example, we see that

$$
\begin{aligned}
& R Q_{i}=\sum_{j=1}^{M} D^{(Q)}(R)_{j i} Q_{j}, \\
& R \frac{\partial V}{\partial Q_{i}}=\sum_{j=1}^{M} D^{(Q)}(R)_{j i} \frac{\partial V}{\partial Q_{j}},
\end{aligned}
$$

where the representation matrix, $D^{(Q)}(R)$, is assumed real and orthogonal. Point groups exist for which complex elements are required in fully reduced representation matrices, but cases of molecules matching these are rare. The present formalism can be extended to that case, if needed. Clearly, the first order term in the Taylor's expansion is invariant to $R$. We assume the $D^{(Q)}(R)$ is completely reduced, i.e., it is in block diagonal form with various of the IRs appearing in the blocks. Therefore, the sums in Eq. (A4) really run over smaller closed sets. The first derivative coefficients divide up into basis sets for IRs in a similar fashion to the $Q$ 's themselves.

$$
R \frac{\partial V}{\partial Q_{n \Gamma i}}=\sum_{j=1}^{g \Gamma} D^{(\Gamma)}(R)_{j i} \frac{\partial V}{\partial Q_{n \Gamma j}}
$$

The foregoing was in the nature of an introduction to the subject we wish to deal with: The symmetry properties of the VC coefficients,

$$
W\left(n_{1} \Gamma_{1} i ; n_{2} \Gamma_{2} j ; n_{3} \Gamma_{3} k\right)=\left\langle\psi_{n_{1} \Gamma_{1^{i}}}\left|\frac{\partial V}{\partial Q_{n_{2} \Gamma_{2} j}}\right| \psi_{n_{3} \Gamma_{3} k}\right\rangle,
$$

which occur in Eqs. (12) and (13). The selection rules for vibrational excitation are inherent in these quantities; we need to know which ones are zero and which ones are not. At the outset we point out that the integrands of the integrals in Eq. (A6) are simple products of factors. In addition, the molecular electronic states may be taken as real, and the $W$ s are therefore also real.

The number of $W$ integrals is important. There are two cases that must be distinguished, depending upon whether $n_{1}=n_{3}$ and $\Gamma_{1}=\Gamma_{3}$. When both of thesc equalities apply, the electronic wave functions in Eq. (A6) are taken from the same set of functions, and

$$
\psi_{n_{1} \Gamma_{1} i} \psi_{n_{1} \Gamma_{1} j}=\psi_{n_{1} \Gamma_{1} j} \psi_{n_{1} \Gamma_{1} i}
$$

because simple multiplication is commutative. Therefore, these two quantities cannot contribute to the basis independently. The total number of $W$ integrals is

$$
\begin{aligned}
& G=\frac{1}{2} g_{\Gamma_{1}}\left(g_{\Gamma_{1}}+1\right) g_{\Gamma_{2}} ; n_{3}=n_{1} ; \Gamma_{3}=\Gamma_{1}, \\
& G=g_{\Gamma_{1}} g_{\Gamma_{2}} g_{\Gamma_{3}} ; \text { otherwise, }
\end{aligned}
$$

in each of the two cases.

In either case the $G$ quantities may be formed into linear combinations that comprise a completely reduced basis for the point group. We call this representation $\Gamma_{W}$. The IRs present in $\Gamma_{W}$ determine whether the $W$ 's are all zero, or whether some of them may be nonzero. The transformation to the reducible form then gives the actual values of the $W$ 's in terms of an irreducible set of integrals. Case 1: $n_{3}=n_{1}$ and $\Gamma_{3}=\Gamma_{1}$. We must determine the IRs present in the $G \times G$ representation supported by the $W$ integrals, or what is the same thing, the integrands in these integrals. For this case we must determine the representation supported by the products of the wave functions. With binary products of functions from the same set, we must use the symmetrized square ${ }^{28}$ of the $\Gamma_{1}$ representation, which is symbolized, $\left[\Gamma_{1}^{2}\right]$. We note that there is another representation that can be constructed from $\Gamma_{1}$, viz., the antisymmetrized square, $\left\{\Gamma_{1}^{2}\right\}$. The characters of these two representations are given from those of the original representation by

$$
\begin{aligned}
& {\left[\chi^{2}\right](R)=\frac{1}{2}\left[\chi(R)^{2}+\chi\left(R^{2}\right)\right],} \\
& \left\{\chi^{2}\right\}(R)=\frac{1}{2}\left[\chi(R)^{2}-\chi\left(R^{2}\right)\right],
\end{aligned}
$$

respectively. The first of these expressions is different from the normal cross product representation only for multidimensional representations. The second is identically zero for one-dimensional representations.

The sum of the symmetrized and antisymmetrized representations is just the normal cross product (square in this case) representation

$$
\left[\Gamma_{1}^{2}\right]+\left\{\Gamma_{1}^{2}\right\}=\Gamma_{1} \times \Gamma_{1} .
$$

But in this case where the binary products of the wave functions satisfy Eq. (A7), the antisymmetrized square is identically zero. Therefore, the binary products support only the remaining part, $\left[\Gamma_{1}^{2}\right]$. Finally we have

$$
{ }_{\underline{-}}{ }_{W}=\left[\Gamma_{1}^{2}\right] \times \Gamma_{2} .
$$

Case 2: $n_{3} \neq n_{1}$ or $\Gamma_{3} \neq \Gamma_{I}$. The details of the symmetrized or antisymmetrized square are not needed in this case, and we have simply

$$
\Gamma_{W}=\Gamma_{1} \times \Gamma_{2} \times \Gamma_{3} .
$$

We must now write the transformation of $\Gamma_{W}$ to a completely reduced form 


$$
\begin{aligned}
\bar{W}_{n \Gamma l}= & \sum_{i j k} C(i j k \mid n \Gamma l) W\left(n_{1} \Gamma_{1} i ; n_{2} \Gamma_{2} j ; n_{3} \Gamma_{3} k\right) \\
& \times \sum_{i j k} C(i j k \mid n \Gamma l) C\left(i j k \mid n^{\prime} \Gamma^{\prime} l^{\prime}\right) \\
= & \delta_{n n^{\prime}} \delta_{\Gamma \Gamma^{\prime}} \delta_{l l^{\prime}} \sum_{n \Gamma l} C(i j k \mid n \Gamma l) C\left(i^{\prime} j^{\prime} k^{\prime} \mid n \Gamma l\right) \\
= & \delta_{i i^{\prime}} \delta_{j j^{\prime}} \delta_{k k^{\prime}} .
\end{aligned}
$$

The only ones of these integrals that can be nonzero are those belonging to the totally symmetric IR of the group. We symbolize this specific representation as $\Gamma_{A}$, and we may write

$$
\bar{W}_{n \Gamma l}=A_{n} \delta_{\Gamma \Gamma_{A}}, \quad n=1,2,3, \ldots, m_{\Gamma_{A}},
$$

where $m_{\Gamma A}$ is the number of times $\Gamma_{A}$ appears in $\Gamma_{W}$, and is the number of possibly independent quantities upon which all of the $W$ 's depend. We may now write the original integrals as

$$
W\left(n_{1} \Gamma_{1} i ; n_{2} \Gamma_{2} j ; n_{3} \Gamma_{3} k\right)=\sum_{n=1}^{m_{\Gamma_{A}}} C\left(i j k \mid n \Gamma_{A} 1\right) A_{n} .
$$

Succeeding terms are more complicated, but may be treated in essentially the same way. We give a brief description of the method used for the second derivative terms. The $M$ normal coordinates, $Q_{i}$, appear as the $M(M+1) / 2$ linearly independent binary products, $Q_{i} Q_{j}$, multiplied by the second derivative terms. The representation supported by the binary products is just the symmetrized square again, $\left[\Gamma_{Q}^{2}\right]$. And, again, the representation supported by the derivatives is the same as that supported by the products of $Q$ 's. The complication in this case is that linear combinations must be formed to obtain IRs. When these linear combinations are combined with wave functions in integrals for the second derivatives symmetry analyses parallel to those we made for the $W$ 's are possible.

The higher order terms are treated the same way except that now we need the symmetrized $n$th power, $\left[\Gamma_{Q}^{n}\right]$. For example, the character for the symmetrized cube is

$$
\left[\chi^{3}\right](R)=\frac{1}{6}\left[\chi(R)^{3}+3 \chi(R) \chi\left(R^{2}\right)+2 \chi\left(R^{3}\right)\right] \text {. }
$$

Murnaghan ${ }^{28}$ discusses the general case. Once the IRs present in the derivatives are determined this way, the symmetry analysis of the corresponding integrals with wave functions is possible.

${ }^{1}$ S. F. Wong and G. J. Schulz, Phys. Rev. Lett. 35, 1429 (1975).

${ }^{2}$ I. C. Walker, A. Stamatovic, and S. F. Wong, J. Chem. Phys. 69, 5532 (1978).

${ }^{3}$ K.-H. Kochem, W. Sohn, K. Jung, H. Ehrhardt, and E. S. Chang, J. Phys. B 18, 1253 (1985).

${ }^{4}$ F. Edard, A. P. Hitchcock, and M. Tronc, J. Phys. Chem. 94, 2768 (1990).

${ }^{5}$ X. Shi, T. M. Stephen, and P. D. Burrow, J. Chem. Phys. 96, 4037 (1992).

${ }^{6}$ A. Mann and F. Linder, J. Phys. B 25, 545 (1992).

${ }^{7}$ A. Mann and F. Linder, J. Phys. B 25, 1621 (1992).

${ }^{8}$ A. Mann and F. Linder, J. Phys. B 25, 1633 (1992).

${ }^{9}$ K.-H. Kochem, W. Sohn, N. Hebel, K. Jung, and H. Ehrhardt, J. Phys. B 18, 4455 (1985).

${ }^{10}$ W. Sohn, K.-H. Kochem, K. M. Scheuerlein, K. Jung; and H. Ehrhardt, J. Phys. B 20, 3217 (1987).

${ }^{11}$ G. A. Gallup, Pliys. Rev. A 34, 2746 (1986); 36, 2485 (1987).

${ }^{12}$ H. Estrada, L. S. Cederbaum, and W. Domcke, J. Chem. Phys. 84, 152 (1986).

${ }^{13}$ M. Ben Arfa and M. Tronc, J. Electron Spectrosc. Relat. Phenom. 50, 117 (1990).

${ }^{14}$ T. Azumi and K. Matsuzaki, Photochem. Photobiol. 25, 315 (1977).

${ }^{15} \mathrm{G}$. Fischer, Vibronic Coupling (Academic, London, 1984).

${ }^{16}$ H. Feshbach, Ann. Phys. (N.Y.) 5, 357 (1958).

${ }^{17}$ U. Fano, Phys. Rev. 124, 1866 (1961).

${ }^{18}$ W. Domcke, Phys. Rep. 108, 97 (1991)

${ }_{1}^{19}$ D. Chen and G. A. Gallup, J. Chem. Phys. 93, 8893 (1990).

${ }^{20}$ See Ref. 12 , this is the term they denote as $W$ in their discussion on page 153, column 2, paragraph 2.

${ }^{21}$ L. Sanche and G. J. Schulz, J. Chem. Phys. 58, 479 (1973).

${ }^{22}$ R. Azria and G. J. Schulz, J. Chem. Phys. 62, 573 (1975).

${ }^{23}$ M. Allan, J. Electron Spectrosc. Relat. Phenom. 48, 219 (1989).

${ }^{24} \mathrm{G}$. Herzberg, Infrared and Raman Spectra of Polyatomic Molecules (Van Nostrand, New York, 1945).

${ }^{25}$ H. A. Jahn and E. Teller, Proc. R. Soc. London, Ser. A 161, 220 (1937); I. B. Bersuker and V. Z. Polinger, Vibronic Interactions in Molecules and Crystals (Springer, Berlin, 1989) give in Appendix C and references therein a modern version of the proof of the Jahn-Teller theorem.

${ }^{26}$ F. H. Read, J. Phys. B 1, 893 (1968).

${ }^{27}$ E. B. Wilson, J. C. Decius, and P. C. Cross, Molecular Vibrations (McGraw-Hill, New York, 1955).

${ }^{28}$ These manipulations are not discussed in a great number of places. See L. D. Landau and E. M. Lifshitz, Quantum Mechanics, Non-Relativistic Theory (Pergamon, Oxford, 1965), Sect. 94, p. 353. A much more complete discussion is given in F. D. Murnaghan, The Theory of Group Representations (Dover, New York, 1963), p. 68f. 